\title{
Electric Cell Substrate Impedance Sensing (ECIS) as a unique technique in cancer metastasis research
}

\author{
Monika Prendecka', Jacek Frankowski ${ }^{2}$, Grzegorz Sobieszek ${ }^{3}$, Lucyna Kapka-Skrzypczak ${ }^{4}$, \\ Aneta Skwarek-Dziekanowska ${ }^{3}$, Teresa Małecka-Massalska' \\ 1 Physiology Department, Medical University, Lublin, Poland \\ ${ }^{2}$ Independent Public Health Care Centre, Radzyń Podlaski, Poland \\ ${ }^{3}$ 1st Military Clinical Hospital, Lublin, Poland \\ ${ }^{4}$ Department of Molecular Biology and Translational Research, Institute of Rural Health, Lublin, Poland
}

Monika Prendecka, Jacek Frankowski, Grzegorz Sobieszek, Lucyna Kapka-Skrzypczak, Aneta Skwarek-Dziekanowska, Teresa MałeckaMassalska. Electric Cell Substrate Impedance Sensing (ECIS) as a unique technique in cancer metastasis research. J Pre-Clin Clin Res. 2018; 12(4): 142-144. doi: 10.26444/jpccr/100616

\begin{abstract}
Introduction. Metastasis is defined as the ability of cancer cells to migrate from the site of primary tumour growth to a distant location. The metastatic tumour is usually more lethal than the initial primary cancer which is the reason for the metastatic cancer being more lethal. This cancer metastasis study gives a very important insight into how invasive cells cross the endothelial and epithelial monolayers. By simultaneously monitoring both barrier function and cell viability, Electric Cell Substrate Impedance Sensing $\left(\mathrm{ECIS}^{\circledR}\right)$ can distinguish between the transmigration mechanisms that leave the monolayer intact from those that disrupt the cell layer.

Objective. The aim of the study is to show the unique (ECIS) technique as aq useful tool in the study of cancer metastasis. Discussion. Intensive research on the method development is the reason for attempts to explain the relationship between electrical changes in cells or on their surface, and the processes influencing survival, which require a holistic approach. As a result of new technical possibilities, including ECIS, and assuming that changes in the electrical properties of cells precede changes on the biochemical level, it would be very interesting to examine the character and dynamics of these changes. The electric parameters measured by the ECIS system are impedance, resistance and capacitance. All of them can be used to examine cell transformation, migration and invasion, cell proliferation and tight junctions as a function of barrier resistance. Conclusions. As cell function modulates cell morphology, ECIS is a method capable of detecting and quantifying for recording morphology changes in the subnanometer to micrometer range. This study describes the possibility of using ECIS in metastasis research.
\end{abstract}

\section{Key words}

cancer metastasis, impedance, ECIS

\section{INTRODUCTION}

Metastasis is the general term used to describe the spread of cancer cells from the primary tumour to surrounding tissues and distant organs. It is the leading cause of cancer mortality, and it is also known that metastasis accounts for about $90 \%$ of deaths from cancer [1]. Metastases resistant to conventional therapy are the leading cause of death due to cancer. Biological heterogeneity of metastases is a serious obstacle in the treatment process. However, the growth and survival of metastases depends on the interaction between the cancer cells and host homeostatic mechanisms. The targeting of these interactions, apart from the action on the tumour cells themselves, may cause a synergistic therapeutic effect against existing metastases. Although the survival rate of cancer patients has significantly increased in recent years, progress is mainly related to the early detection of cancer and inhibition of disease progression. Progress in the treatment of cancer metastasis is unfortunately still insufficient.

Address for correspondence: Monika Prendecka, Physiology Department, Medical University, Lublin, Poland

E-mail:m.prendecka@gmail.com

Received: November 2018; Accepted: December 2018
The process of metastases includes a sequence of interrelated steps. In the final of this cascade reaction, the cancer cells must separate from the primary tumour, enter the circulatory and lymphatic systems, avoid the attack of the immune system, extravasation in distant capillary bearings, and in the end invade and proliferate in distant organs. The first step leading to the final in the form of meta cells in distant organs is always the same - the transformation of a healthy, primary cell into an "immortal" form of cancer. It is extremely difficult to study cellular transformation by means of traditional assays, e.g. chemical staining or optical examination. In the early stages of transformation, chemical assays are time consuming and provide only a static endpoint, resulting in the destruction of the cell. Electric cell substrate impedance sensing (ECIS) is the measurement of resistance and reactance across an electrode containing growing cells which make it great tool in studying of cancer metastasis. The impedance method seems to be a promising, noninvasive technique for detection of the whole cellular behaviour and morphology changes.

The pioneers of this technique were Giaever and Keese who developed electric cell-substrate impedance sensing (ECIS) as a continuous monitoring system for studying cell behaviour, including proliferation, adhesion, motility and 
morphological changes, using a noninvasive, real-time, and label-free method [2]. ECIS is a useful predictor of cellular transformation because it sensitively measures many of cellular characteristics that change during transformation [3]. The direct parameters derived from impedance measurements are the resistance and capacitance of cells. The quality and function of the cell barrier is represented by the resistance, and therefore the resistance towards para- and trans-cellular current flow should be considered. Capacitance provides an overall measure of electrode coverage. Therefore, the different behaviour of the cells after the seeding, adherence, proliferation and their reaction to substances added to the substrate as a treatment produce change in impedance.

Basics of the ECIS method. These measurements detect changes in the morphology of the cells which, in turn, are derived from specific metabolic processes. In this system, electrodes are used which apply low alternating current and electrodes to measure the voltage change mounted at the bottom of a standard matrix. Impedance is measured according to Ohm's law, $\mathrm{Z}=\mathrm{V} / \mathrm{l}$. When the cells attach to the matrix and electrodes they act as insulators increasing impedance. The flow of the current is hindered depending on the number of cells covering the electrode, shape of the cells, and type of cells attached to the electrode surface.

The structure design of ECIS measuring system includes: two electrodes (one is a small working electrode and the other a large counter electrode on the bottom of the culture plate) connected to the edge of a culture chip, and the chip connected to a lock-in amplifier. The whole setup is placed inside an incubator in steady conditions $37^{\circ} \mathrm{C}$ and under a 5\% $\mathrm{CO}_{2}$ atmosphere. After cell seeding, the cells drift downward and attach to the stratum of the electrode, which then passes the impeded current directly into the bulk electrolyte as the result of anchored plasma membrane intrusion above the electrode surface [4].

During cell stimulation they change their function, and the accompanying changes in their morphology alters impedance. The generated data is impedance in time. In this system, frequency is a very important factor: at low frequencies $(<2,000 \mathrm{~Hz})$ the majority of the current flows between the cells, while at high frequencies $(>40,000 \mathrm{~Hz})$, most of the current flows directly through the isolated cell membranes. The impedance at high frequencies is affected more significantly by the cell membrane, while at low frequencies the response is more dependent on the space below and between cells.

Electric parameters measurements with ECIS. ECIS is an advanced in vitro impedance measuring system using alternating current $(\mathrm{AC})$ to determinate the behaviour of cells in physiological conditions. Cell function modulates cell morphology. ECIS is capable of detecting and quantifying morphology changes in the subnanometer to micrometer range. In ECIS, a small alternating current (I) is applied across the electrode pattern at the bottom of the ECIS arrays (direct current DC cannot be used). This results in a potential (V) across the electrodes which is measured by the ECIS instrument. As cells grow and cover the electrodes, the current is impeded in a manner related to the number of cells covering the electrode, the morphology of the cells and the nature of the cell attachment. When cells are stimulated to change their function, the accompanying changes in cell morphology alter the impedance. The data generated is impedance versus time.

Ohm's law is the basis for the measurement of the electrical impedance of biological objects, which describes the relation between resistance (R), current (I) and voltage (U) in an electrical circuit at a given time $(\mathrm{t})$. In $\mathrm{DC}$ circuit: $\mathrm{R}(\mathrm{t})=$ $\mathrm{U}(\mathrm{t}) / \mathrm{I}(\mathrm{t})$. However, it should be noted, that working in the AC system, current and voltage not only differ in their amplitude, but also in their phase $(\varphi)$. In this case, resistance alone is not sufficient to describe these relations. Instead, the complex impedance $(\mathrm{Z})$, or in most cases the magnitude of the impedance $(|\mathrm{Z}|)$ are used, containing resistance plus reactance $(\mathrm{X})$, which results from $\mathrm{AC}$ flow through capacitors and inductors driving the phase shift between voltage and current [5].

Impedance changes reflect cell transformation. Growing cells often act as insulating particles because their plasma membrane interferes with the free space immediately above the electrode for current flow. This produces a drastic change in the measured impedance due to cell attachment and spreading. As the cells die, the decrease in impedance value is recorded in contrast to living cells. The membranes of the dying cells disintegrate and no longer act as barriers to current. Immortalization of cells increases impedance; the resistance value between electrodes also increases as the cells grow and proliferate.

Besides coverage of the electrode, the thickness of the cellular layer, cell-cell and cell-electrode adhesions are also factors in ECIS measurement [3]. Different cell types also vary in the strenght of their cell-cell adhesion. A very tightly packed monolayer with strong focal adhesions between the cells results in significantly higher impedance than those that form a loosely packed layer, i.e. as long as all other parameters are constant $[6,7,8]$. The increase in cell adhesion and packing density, and hence increase in the impedance, may indicate cell transformation.

Metastasis - migration and invasion in ECIS measurements. The most characteristic cancer metastatic ability is migration and invasion. The ECIS instrument uses mili-Ampere currents in order to generate 1 - 10 volt potentials across the cell monolayer. This potential across the cell monolayer is sufficient to induce pores in the cytoplasmic membrane. If this pulse is applied in a brief pulse $(<0.5 \mathrm{sec})$, then the effect is to reversibly electroporate the cells. If the pulse is big enough ( $>1 \mathrm{~V}$ across the cell membrane) or sustained $(\sim>$ 5 seconds) then the cells die by irreversible electroporation. This results in a very defined area of dead cells, and a resulting drop in impedance. Then cells along the periphery of the electrode then migrate to repopulate the electrode, healing the electrically-induced wound. This recovery after electrical wounding is the ECIS Cell Migration Assay [9].

Daouti et al. [10] used the ECIS technique for demonstrating the efficacy of trienopyridone as the migration specific inhibitor of oncolytic phophatase (PRL-3). They showed that migration was inhibited, but not cellular proliferation. In another experiment, ECIS was used to demonstrate the decrease of migration of glioblastomas after paxilline treatment. Glioblastomas switch from migration to a more invasive/metastatic phenotype upon exposure to sub-lethal irradiation, which can be minimized using paxilline [11,12]. These studies showed that ECIS can distinguish between 
attachment and migration in various types of cells. Keese et al. studied migration of cells through a monolayer and an artificial cell culture system for this purpose [13]. By using prostate cell lines placed over the top of HUVEC cells, a decrease was found in impedance during migration of the cells through the endothelial HUVEC monolayer. After comparison of impedance measurements of more metastatic and less metastatic cell lines, Keese et al. also showed that impedance correlated with metastatic potential. This led the researchers to the conclusion that ECIS can be used to determine the metastatic ability of a biopsy which, in turn, could be used as a factor in recommending a treatment course [3].

ECIS in monitoring of cell proliferation. As cells proliferate, the impedance is affected by cell number and cell morphology. In most cases, cells grow asynchronously and impedance grows gradually to reach the point where the cells become confluent. Linear change in the impedance value correlated with cell number can be observed while cells are sub-confluent. Substances affecting cell growth can be introduced before or after the cells have become attached, to distinguish changes in growth from the ability of cells to attach to the substrate.

ECIS is capable of monitoring the proliferation and death of anchorage-dependent mammalian cells in vitro continuously, without using any kind of label. Instead of counting viable cells at different time points of an experiment to estimate the rate of cell division or cell death by classical means, ECIS reads the coverage of gold-film electrodes deposited on the bottom of the culture dish with non-invasive AC voltages [14].

Study of tight junctions in cancer metastasis. Tight junctions play a vital role in maintaining cell-to-cell integrity. Loss of adhesion of the tight junction structure can lead to invasion and ultimately to the metastasis of cancer cells [15]. Tight junctions are the first barrier that cancer cells must overcome in order to metastasize. The use of ECIS to moniotor was first demonstrated by Tiruppathi et al. [16] using bovine endothelial cells.

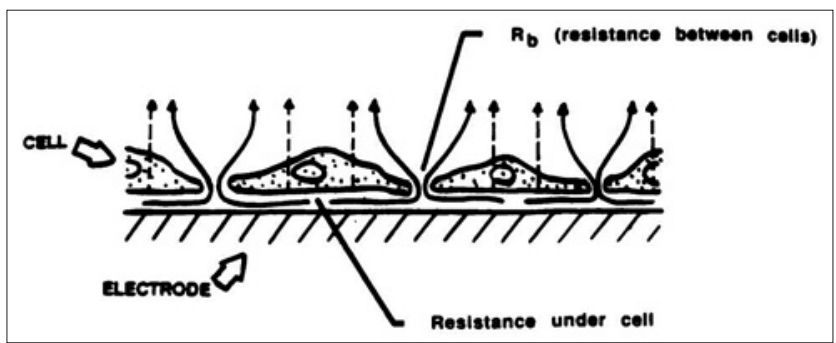

Figure 1. Cells in tissue culture. Calculated resistance is due to the current flow under the cells and additional resistance because the current must flow out between the cells. Broken line represents capacitive current flow through the cell membranes [17]

As shown by Giaever and Keese (1993) (Fig. 1) [17], by using the ECIS model the true barrier function due to changes in intracellular junctions can be calculated. In the model, the resistance and capacitance of the cell free electrode is measured at several different frequencies. Cells are treated as disc shapes that hover a small distance above the electrode, have an insulating membrane and filled with conducting electrolyte [15]. In this arrangement, resistance and capacitance of the gold electrode remains unchanged, but the changes in electrical parameters are recorded due the cells altering the flow or current. In this system, some current will flow through the constricted space beneath the cells (between the cells and the electrode), while some current will flow through the spaces between the cells, which is termed the barrier resistance.

As may be concluded, in the future ECIS could be used to develop anticancer treatment to a high degree of "personalization" where anticancer compounds are tested on an individual's cancer cells to design personalized treatment.

\section{REFERENCES}

1. Chaffer CL, Weinberg RA. A perspective on cancer cell metastasis. Science. 2011; 331(6024): 1,559-64.

2. Giaever I, Keese CR. Monitoring fibroblast behavior in tissue culture with an applied electric field. Proc Natl Acad Sci. 1984; 81:3761-3764.

3. Plugner B, Choi ChK, Sparer TE. Electrical Cell-Substrate Impedance Sensing for Measuring Cellular Transformation, Migration, Invasion, and Anticancer Compound Screening. Cancer Metastasis - Biology and Treatment, Electrical Cell-Substrate Impedance Sensing and Cancer Metastasis. 2012; 17: 55-69.

4. Giaever I, Keese CR. A morphological biosensor for mammalian cells. Nature. 1993; 366 (6455): 591-592.

5.Pänke O, Balkenhohl T, Kafka J, Schäfer D, Lisdat F. Impedance spectroscopy and biosensing. Adv Biochem Eng Biotechnol. 2008; 109 (11), 195237.

6. Choi CK, English AE, Kihm KD, Margraves CH. Simultaneous dynamic optical and electrical properties of endothelial cell attachment on indium tin oxide bioelectrodes. J Biomed Opt. 2007; 12(6): 064028.

7. Choi CK, English AE, Jun S-I, Kihm KD, Rack PD. An endothelial cell compatible biosensor fabricated using optically thin indium tin oxide nitride electrodes. Biosens Bioelectron. 2007; 22: 2585-2590.

8. Choi CK, Sukhthankar M, Kim C-H, Lee S-H, English A, Kihm KD, et al. Cell adhesion property affected by cyclooxygenase and lipoxygenase: opto-electric approach. Biochem Biophys Res Commun. 2010; 391: 1385-1389.

9. Applied Biophysics 1-866-301-ECIS (3247), web- biophysics.com

10. Bosserhoff AK, Ellmann L, Kuphal S. Melanoblasts in culture as an in vitro system to determine molecular changes in melanoma. John Wiley \& Sons A/S, Exp Dermatol. 2011; 20: 435-440.

11. Wild-Bode C, Weller M, Rimner A, Dichgans J, Wick W. Sublethal irradiation promotes migration and invasiveness of glioma cells. Cancer Res. 2001; 61: 2744-2750.

12. Park C-M, Park MJ, Kwak HJ, Lee HC, Kim MS, Lee SH, et al. Ionizing radiation enhances matrix metalloproteinase- 2 secretion and invasion of glioma cells through Src/epidermal growth factor receptor-mediated p38/Akt and phosphatidylinositol 3-kinase/Akt signaling pathways. Cancer Res. 2006; 66(17): 8511-8529.

13. Keese CR, Bhawe K, Wegener J, Giaever I. Real-time impedance assay to follow the invasive activities of metastatic cells in culture. Biotechniques. 2002; 33(4):842-844, 846, 848-850.

14. Stolwijk JA, Michaelis S, Wegener J. Cell Growth and Cell Death Studied by Electric Cell-Substrate Impedance Sensing. Cell Growth and Cell Death Studied by Electric Cell-Substrate Impedance Sensing. In: Jiang W. (eds) Electric Cell-Substrate Impedance Sensing and Cancer Metastasis. Cancer Metastasis - Biology and Treatment. 2012; 17: 85-117.

15. Martin TA, Jiang WG. Tight Junctions in Cancer Metastasis, Cancer Metastasis - Biology and Treatment, Electrical Cell-Substrate Impedance Sensing and Cancer Metastasis. 2012; 17: 119-130.

16. Tiruppathi Ch, Malik AB, Del Vecchio PJ, Keeset ChR, Giaevert I. Electrical method for detection of endothelial cell shape change in real time: Assessment of endothelial barrier function. Proc Nati Acad Sci. 1992; 89: 7919-7923.

17. Giaever I, Keese CR. Micromotion of mammalian cells measured electrically. Proc Natl Acad Sci. 1993; 88(17): 7896-7900. Erratum in: Proc Natl Acad Sci USA 1993; 90(4):1634. 